\title{
Charged Higgs in view of the LHC constraints in phenomenological MSSM
}

\author{
Farvah Mahmoudi* \\ CERN Theory Division, CH-1211 Geneva 23, Switzerland \\ Clermont Université, Université Blaise Pascal, CNRS/IN2P3, LPC, BP 10448, F-63000 \\ Clermont-Ferrand, France \\ E-mail: mahmoudiein2p3.fr
}

\author{
Alexandre Arbey \\ Centre de Recherche Astrophysique de Lyon, Observatoire de Lyon, Saint-Genis Laval Cedex, \\ F-69561, France; CNRS, UMR 5574; Ecole Normale Supérieure de Lyon, Lyon, France; \\ Université de Lyon, France; Université Lyon 1, F-69622 Villeurbanne Cedex, France \\ CERN Theory Division, CH-1211 Geneva 23, Switzerland \\ E-mail: alexandre.arbeydens-lyon.fr
}

\section{Marco Battaglia}

Santa Cruz Institute of Particle Physics, University of California, Santa Cruz, CA 95064, USA;

Lawrence Berkeley National Laboratory, Berkeley, CA 94720, USA

CERN, CH-1211 Geneva 23, Switzerland

E-mail: marco.battagliaecern.ch

We discuss the consequences of the observation of a light Higgs boson with the mass and rates reported by the ATLAS and CMS collaborations on the parameter space of the phenomenological MSSM, including also the LHC searches for heavier Higgs bosons and supersymmetric particles, as well as the constraints from $B$ physics and dark matter for the charged Higgs boson.

Prospects for Charged Higgs Discovery at Colliders

October 8-11, 2012

Uppsala University Sweden

\footnotetext{
* Speaker.
} 


\section{Introduction}

Charged Higgs bosons are important ingredients in theories with more than one Higgs doublet such as supersymmetry (SUSY), since the observation of a fundamental charged scalar would provide unambiguous evidence for an extended Higgs sector. Before the start of the LHC experiment, the expectation for direct discovery of SUSY particles was very high. After two years of LHC running, we now have evidence for a neutral boson compatible with the Standard Model (SM) Higgs boson, but no signal of SUSY particles has been detected and therefore strong limits on the masses of strongly interacting particles have been obtained. However, most of the analyses have been performed in highly constrained MSSM scenarios, such as CMSSM. Here we will instead discuss the phenomenology of the charged Higgs boson in view of the LHC constraints in the more general and unconstrained set-up of the phenomenological MSSM. We will also show that while direct searches are pushing the masses to larger values, which is not enough to confirm or exclude SUSY, the information from the Higgs sector constitute an alternative and powerful path to constrain SUSY efficiently.

\section{Phenomenological MSSM}

To study the implication of the LHC Higgs and SUSY searches we consider the unconstrained phenomenological MSSM (pMSSM) with 19 parameters [1]. Most of the previous studies considered the highly constrained models with a small number of free parameters. However, these models are not representative of a generic MSSM scenario where the particle mass parameters are independent. As we will see below, the results can be very different in such generic scenarios.

To explore the pMSSM, we perform a flat scan over the parameters in the ranges given in Table 1. The particle spectra are generated for more than 100M points using SOFTSUSY [2] and SUSPECT [3]. We impose the SUSY and Higgs mass limits from LEP and Tevatron as described in [4]. The flavour observables, muon anomalous magnetic moment and relic density are computed with SuperIso Relic [5], and we apply the constraints given in Table 2. We do not address here the consequences of the dark matter direct detection results which are discussed thoroughly in [6]. To evaluate the consequences of the SUSY searches, we compute the supersymmetric particle decay rates with SDECAY [7] and we use PYTHIA 6 [8] for event generation of inclusive SUSY production in $p p$ interactions. The generated events are then passed through fast detector simulation using Delphes [9]. The Higgs decay rates are computed with HDECAY [10] and the gluon fusion and VBF cross sections of the lightest CP-even Higgs with HIGLU [11] and FeynHiggs [12]. More details can be found in [4, 13].

\section{Constraints from the lightest Higgs boson mass and decay rates}

Another way to efficiently constrain SUSY is using the information from the Higgs sector. In the following, we consider that the new boson discovered at the LHC corresponds to the lightest $\mathrm{CP}$-even Higgs boson. The signal strength, $\mu$, is defined as:

$$
\mu_{X X}=\frac{\sigma(p p \rightarrow h) \mathrm{BR}(h \rightarrow X X)}{\sigma(p p \rightarrow h)_{\mathrm{SM}} \mathrm{BR}(h \rightarrow X X)_{\mathrm{SM}}}
$$




\begin{tabular}{|c|c||c|c|}
\hline Parameter & Range & Parameter & Range \\
\hline \hline $\tan \beta$ & {$[1,60]$} & $M_{\tilde{e}_{L}}=M_{\tilde{\mu}_{L}}$ & {$[50,2500]$} \\
\hline$M_{A}$ & {$[50,2000]$} & $M_{\tilde{e}_{R}}=M_{\tilde{\mu}_{R}}$ & {$[50,2500]$} \\
\hline$M_{1}$ & {$[-2500,2500]$} & $M_{\tilde{\tau}_{L}}$ & {$[50,2500]$} \\
\hline$M_{2}$ & {$[-2500,2500]$} & $M_{\tilde{\tau}_{R}}$ & {$[50,2500]$} \\
\hline$M_{3}$ & {$[50,2500]$} & $M_{\tilde{q}_{1 L}}=M_{\tilde{q}_{2 L}}$ & {$[50,2500]$} \\
\hline$A_{d}=A_{s}=A_{b}$ & {$[-10000,10000]$} & $M_{\tilde{q}_{3 L}}$ & {$[50,2500]$} \\
\hline$A_{u}=A_{c}=A_{t}$ & {$[-10000,10000]$} & $M_{\tilde{u}_{R}}=M_{\tilde{c}_{R}}$ & {$[50,2500]$} \\
\hline$A_{e}=A_{\mu}=A_{\tau}$ & {$[-10000,10000]$} & $M_{\tilde{t}_{R}}$ & {$[50,2500]$} \\
\hline$\mu$ & {$[-1000,2000]$} & $M_{\tilde{d}_{R}}=M_{\tilde{s}_{R}}$ & {$[50,2500]$} \\
\hline & & $M_{\tilde{b}_{R}}$ & {$[50,2500]$} \\
\hline
\end{tabular}

Table 1: SUSY parameter ranges (in GeV when applicable).

\begin{tabular}{|c|}
\hline $2.16 \times 10^{-4}<\mathrm{BR}\left(B \rightarrow X_{s} \gamma\right)<4.93 \times 10^{-4}$ \\
\hline $\mathrm{BR}\left(B_{s} \rightarrow \mu^{+} \mu^{-}\right)<5.0 \times 10^{-9}$ \\
\hline $0.56<\mathrm{R}(B \rightarrow \tau \nu)<2.70$ \\
\hline $4.7 \times 10^{-2}<\mathrm{BR}\left(D_{s} \rightarrow \tau \nu\right)<6.1 \times 10^{-2}$ \\
\hline $2.9 \times 10^{-3}<\mathrm{BR}\left(B \rightarrow D^{0} \tau v\right)<14.2 \times 10^{-3}$ \\
\hline $0.985<\mathrm{R}_{\mu 23}(K \rightarrow \mu \nu)<1.013$ \\
\hline$-2.4 \times 10^{-9}<\delta a_{\mu}<4.5 \times 10^{-9}$ \\
\hline
\end{tabular}

Table 2: Low energy constraints applied in our analysis.

\begin{tabular}{|c|c|c|}
\hline Parameter & Value & Experiment \\
\hline$M_{h}$ & $126 \pm 2 \mathrm{GeV}$ & ATLAS + CMS \\
\hline$\mu_{\gamma \gamma}$ & $1.71 \pm 0.26$ & ATLAS + CMS \\
$\mu_{Z Z}$ & $0.97 \pm 0.26$ & ATLAS + CMS \\
$\mu_{W W}$ & $0.85 \pm 0.23$ & ATLAS + CMS \\
\hline$\mu_{b \bar{b}}$ & $1.28 \pm 0.45$ & ATLAS + CMS + (CDF + D0) \\
$\mu_{\tau \tau}$ & $0.71 \pm 0.42$ & ATLAS + CMS \\
\hline
\end{tabular}

Table 3: Higgs signal strengths [14].

and the combination of the Higgs search results presented by ATLAS and CMS are given in Table 3. All the decay rates at present are compatible with those of the SM Higgs boson, with a possible enhancement in the diphoton channel compared to the SM value.

The leading order couplings of the MSSM Higgs bosons can be written in terms of $\beta$ and the Higgs mixing angle:

$$
\alpha=\frac{1}{2} \arctan \left(\tan (2 \beta) \frac{M_{A}^{2}+M_{Z}^{2}}{M_{A}^{2}-M_{Z}^{2}}\right) .
$$

The tree level couplings are given in Table 4. It should be mentioned that higher order corrections 


\begin{tabular}{|c|c|c|c|}
\hline$\phi$ & $g_{\phi u \bar{u}}$ & $g_{\phi d \bar{d}}=g_{\phi \ell \bar{\ell}}$ & $g_{\Phi V V}$ \\
\hline$h$ & $\cos \alpha / \sin \beta \rightarrow 1$ & $-\sin \alpha / \cos \beta \rightarrow 1$ & $\sin (\beta-\alpha) \rightarrow 1$ \\
\hline$H$ & $\sin \alpha / \sin \beta \rightarrow \cot \beta$ & $\cos \alpha / \cos \beta \rightarrow \tan \beta$ & $\cos (\beta-\alpha) \rightarrow 0$ \\
\hline$A$ & $\cot \beta$ & $\tan \beta$ & 0 \\
\hline
\end{tabular}

Table 4: Higgs couplings relative to the SM ones. The arrow indicates the values in the decoupling limit $\left(M_{A} \gg M_{Z}\right)$.

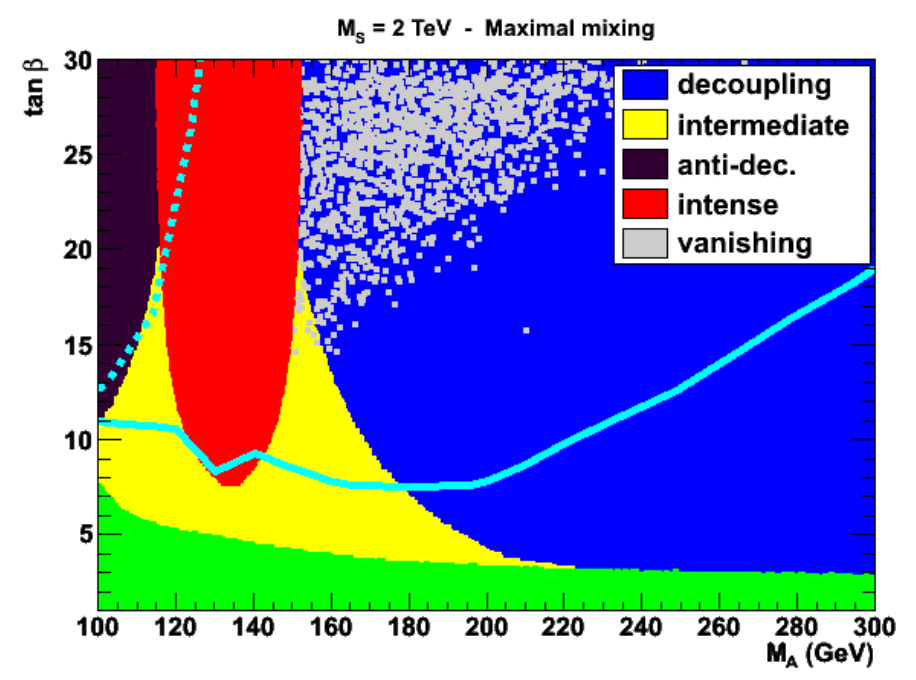

Figure 1: The parameter space for the various regimes of the MSSM Higgs sector in the $\left(M_{A}, \tan \beta\right)$ plane, in the maximal mixing scenario with $M_{S}=2 \mathrm{TeV}$. The upper limit constraints from $A / H \rightarrow \tau^{+} \tau^{-}$[15] (continuous light blue line) and $t \rightarrow H^{+} b$ [16] (dashed blue line) searches at the LHC are shown together with the LEP2 excluded region (green area).

to the tree level couplings can be large for light SUSY particles. For the lightest CP-even $h$, the $g_{h b b}$ is in general the most important coupling. As a result, in the specific SUSY scenarios where this coupling is decreased, the branching fractions to other particles, such as $W W / Z Z$ or $\gamma \gamma$ are in turn enhanced, while $\mathrm{BR}(h \rightarrow b \bar{b})$ is depleted. At tree level the charged Higgs mass is related to the $A$ mass by $M_{H^{ \pm}}^{2}=M_{A}^{2}+M_{W}^{2}$.

In Fig. 1 we present the various regimes of the pMSSM Higgs sector [17]. We start with the decoupling regime, where the light Higgs has SM-like couplings (i.e. $\cos ^{2}(\beta-\alpha)$ is small, corresponding to a vanishing coupling of the $H$ to vector bosons), which occurs at large $M_{A} \approx M_{H^{ \pm}}$ $(\gtrsim 300 \mathrm{GeV}$ ) and $h$ reaches its maximal value. In contrary, in the anti-decoupling regime, the heavy CP-even Higgs has SM like couplings, it occurs for small $M_{A}$, and we have $M_{A} \approx M_{h}<$ $M_{H}, M_{H^{ \pm}}$. The couplings of the $h$ are suppressed in this case, and it can escape the LEP limits. The intermediate regime is between both previous regimes, with intermediate values for $M_{A}$ and $M_{H^{ \pm}}$. In the intense coupling regime on the other hand, the couplings of the $h$ to $b b$ and $\tau \tau$ are enhanced. It occurs at small $M_{A}$ when $M_{h} \approx M_{A} \approx M_{H}$. Finally in the vanishing regime, the couplings of the $h$ to $b b$ or $W W / Z Z$ are very small. This regime occurs generally at large $\tan \beta$ when $\alpha$ is very small 

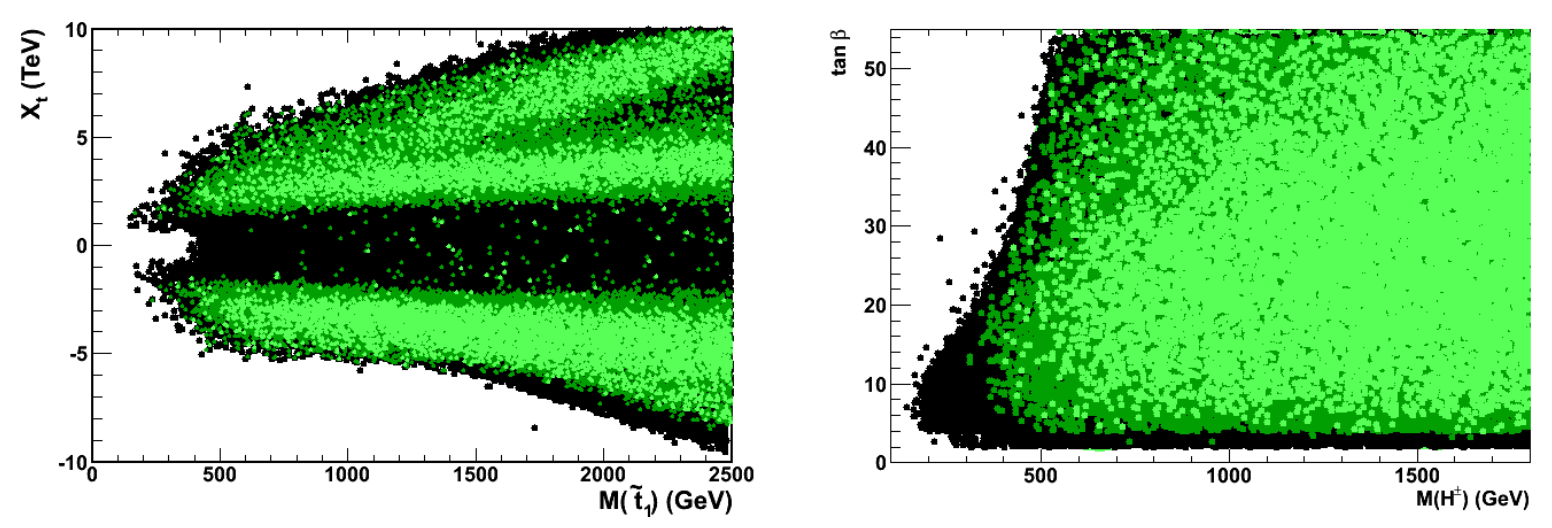

Figure 2: Distributions of the pMSSM points in the $\left(M_{\tilde{t}_{1}}, X_{t}\right)$ and $\left(M_{H^{ \pm}}, \tan \beta\right)$ parameter planes. The black dots show the accepted pMSSM points, those in light (dark) grey the same points compatible at $68 \%(90 \%)$ C.L. with the Higgs constraints of Table 3.

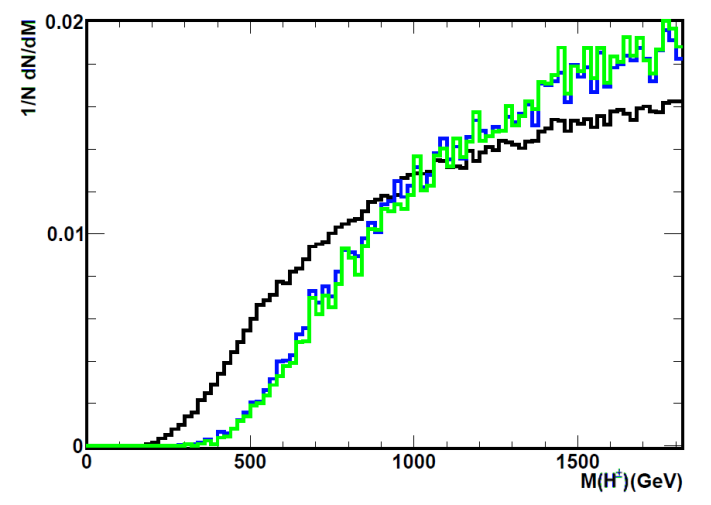

Figure 3: The normalised distribution of the values of $M_{H^{ \pm}}$for the selected pMSSM points (black line) compared to the probability density function obtained from the $\chi^{2}$ probability using $M_{h}, \mu_{\gamma \gamma}$ and $\mu_{Z Z}$ (blue line), as well as $\mu_{\tau \tau}$ and $\mu_{b b}$ (green line).

(at tree level) or when there is a cancellation between tree level terms and radiative corrections due to SUSY loops. As can be seen from the figure, the $A / H \rightarrow \tau^{+} \tau^{-}$limit disfavours the vanishing, intense, and anti-decoupling regimes, and favours the decoupling regime.

In Fig. 2 we present the distribution of pMSSM points compatible with the $h$ boson mass and the observed yields, in the $\left(M_{\tilde{t}_{1}}, X_{t}\right)$ and $\left(M_{H^{ \pm}}, \tan \beta\right)$ parameter planes [14]. To achieve this, we combined all the constraints in Table 3 with a $\chi^{2}$ combination. We notice first that small values of $\left|X_{t}\right|$ are clearly disfavoured, and that stop masses as low as $400 \mathrm{GeV}$ are still compatible with the data. This is mainly the results of the Higgs mass measurement which calls for non minimal mixing in the stop sector. Second, $M_{H^{ \pm}} \lesssim 350 \mathrm{GeV}$ values are strongly disfavoured by the Higgs mass and rate measurements for any value of $\tan \beta$, and therefore the decoupling regime seems again to be favoured by the data.

The distribution of $M_{H^{ \pm}}$is presented in Fig. 3, where each pMSSM point enters with a weight 
equal to its $\chi^{2}$ probability. The probability weighted distribution obtained from this analysis are compared to the normalised frequency distribution for the same observables obtained for accepted points within the allowed mass region $122.5<M_{h}<127.5 \mathrm{GeV}$. We observe a significant suppression of pMSSM points with the charged Higgs boson mass below $450 \mathrm{GeV}$. This is due to the combined effect of the $A / H \rightarrow \tau^{+} \tau^{-}$direct searches and $B_{s} \rightarrow \mu^{+} \mu^{-}$rate, which constrain the light $M_{A}$ and $M_{H^{ \pm}}$and by the suppression of the non-decoupling regime.

\section{Conclusions}

The implications of the Higgs boson discovery by the ATLAS and CMS collaborations for the MSSM have been outlined. The study has been based on broad scans over the pMSSM parameter space where points have been preselected based on constraints from electroweak and flavour physics, dark matter and searches at LEP2, Tevatron and the LHC. We showed that the Higgs searches have impressive impacts on the SUSY parameters. The present data tend to disfavour the possibility of a light charged Higgs boson. However, more data will be needed to draw decisive conclusions.

\section{References}

[1] A. Djouadi et al. [MSSM Working Group Collaboration], hep-ph/9901246.

[2] B. C. Allanach, Comput. Phys. Commun. 143 (2002) 305 [hep-ph/0104145].

[3] A. Djouadi, J.L. Kneur and G. Moultaka, Comput. Phys. Commun. 176 (2007) 426 [hep-ph/0211331].

[4] A. Arbey, M. Battaglia and F. Mahmoudi, Eur. Phys. J. C 72 (2012) 1906 [arXiv:1112.3032].

[5] F. Mahmoudi, Comput. Phys. Commun. 178 (2008) 745 [arXiv:0710.2067]; Comput. Phys. Commun. 180 (2009) 1579 [arXiv:0808.3144]; A. Arbey and F. Mahmoudi, Comput. Phys. Commun. 181 (2010) 1277 [arXiv:0906.0369].

[6] A. Arbey, M. Battaglia and F. Mahmoudi, Eur. Phys. J. C 72 (2012) 2169 [arXiv:1205.2557].

[7] M. Muhlleitner, A. Djouadi and Y. Mambrini, Comput. Phys. Commun. 168 (2005) 46 [hep-ph/0311167].

[8] T. Sjostrand, S. Mrenna and P. Z. Skands, JHEP 0605 (2006) 026 [hep-ph/0603175].

[9] S. Ovyn, X. Rouby and V. Lemaitre, arXiv:0903.2225 [hep-ph].

[10] A. Djouadi, J. Kalinowski and M. Spira, Comput. Phys. Commun. 108 (1998) 56 [hep-ph/9704448].

[11] M. Spira, hep-ph/9510347.

[12] S. Heinemeyer, W. Hollik and G. Weiglein, Comput. Phys. Commun. 124 (2000) 76 [hep-ph/9812320].

[13] A. Arbey, M. Battaglia and F. Mahmoudi, Eur. Phys. J. C 72 (2012) 1847 [arXiv:1110.3726].

[14] A. Arbey, M. Battaglia, A. Djouadi and F. Mahmoudi, Phys. Lett. B 720 (2013) 153 [arXiv:1211.4004].

[15] S. Chatrchyan et al. [CMS Collaboration], Phys. Lett. B 713 (2012) 68 [arXiv:1202.4083].

[16] G. Aad et al. [ATLAS Collaboration], JHEP 1206 (2012) 039 [arXiv:1204.2760].

[17] A. Arbey, M. Battaglia, A. Djouadi and F. Mahmoudi, JHEP 1209 (2012) 107 [arXiv:1207.1348]. 